\title{
The association of rectal equivalent dose in 2 Gy fractions (EQD2) to late rectal toxicity in locally advanced cervical cancer patients who were evaluated by rectosigmoidoscopy in Faculty of Medicine, Chiang Mai University
}

Ekkasit Tharavichtikul, MD', Pooriwat Meungwong, MD², Taned Chitapanarux, MD³, Somvilai Chakrabandhu, MD', Pitchayaponne Klunklin, MD', Wimrak Onchan, MD', Somsak Wanwilairat, PhD', Patrinee Traisathit, $\mathrm{PhD}^{4}$, Razvan Galalae, MD, PhD ${ }^{5}$, Imjai Chitapanarux, MD

'Division of Therapeutic Radiology and Oncology, Department of Radiology, Faculty of Medicine, Chiang Mai University, Chiang Mai, Thailand; ${ }^{2}$ Lampang Cancer Hospital, Lampang, Thailand; ${ }^{3}$ Department of Medicine, Faculty of Medicine, Chiang Mai University, Chiang Mai, Thailand; ${ }^{4}$ Biostatistics and Applied Statistics Laboratory, Department of Statistics, Faculty of Science, Chiang Mai University, Chiang Mai, Thailand;

${ }^{5}$ Faculty of Medicine, Christian-Albrechts University at Kiel, Kiel, Germany

Purpose: To evaluate association between equivalent dose in 2 Gy (EQD2) to rectal point dose and gastrointestinal toxicity from whole pelvic radiotherapy (WPRT) and intracavitary brachytherapy (ICBT) in cervical cancer patients who were evaluated by rectosigmoidoscopy in Faculty of Medicine, Chiang Mai University.

Materials and Methods: Retrospective study was designed for the patients with locally advanced cervical cancer, treated by radical radiotherapy from 2004 to 2009 and were evaluated by rectosigmoidoscopy. The cumulative doses of WPRT and ICBT to the maximally rectal point were calculated to the EOD2 and evaluated the association of toxicities.

Results: Thirty-nine patients were evaluated for late rectal toxicity. The mean cumulative dose in term of EQD2 to rectum was 64.2 Gy. Grade 1 toxicities were the most common findings. According to endoscopic exam, the most common toxicities were congested mucosa (36 patients) and telangiectasia (32 patients). In evaluation between rectal dose in EQD2 and toxicities, no association of cumulative rectal dose to rectal toxicity, except the association of cumulative rectal dose in EQD2 >65 Gy to late effects of normal tissue (LENT-SOMA) scale $\geq$ grade 2 ( $p=0.022$; odds ratio, 5.312; 95\% confidence interval, 1.269-22.244).

Conclusion: The cumulative rectal dose in EOD2 >65 Gy have association with $\geq$ grade 2 LENT-SOMA scale.

Keywords: Cervical cancer, Radiotherapy, Cumulative dose, Rectum, Toxicity, Association

Received 25 February 2014, Revised 4 April 2014, Accepted 22 May 2014.

Correspondence: Ekkasit Tharavichitkul, MD, Division of Therapeutic Radiology and Oncology, Department of Radiology, Faculty of Medicine, Chiang Mai University, Chiang Mai, 239 Huay Kaew Road, Muang District, Chiang Mai 50200, Thailand. Tel: +66-53945456, Fax: +66-53945491, E-mail: paan_31@hotmail.com

(c) This is an Open Access article distributed under the terms of the Creative Commons Attribution Non-Commercial License (http://creativecommons.org/ licenses/by-nc/3.0/) which permits unrestricted non-commercial use, distribution, and reproduction in any medium, provided the original work is properly cited.

www.e-roj.org 


\section{Introduction}

Cervical cancer is the second most common cancer in incidence and mortality in Thai women. In Thailand, the age-standardized rates were 24.5 and 12.8 in incidence and mortality, respectively [1]. Concurrent chemotherapy and radiotherapy is the treatment of choice for locally advanced cancers because combination of these two modalities improves tumor control, overall survival, and progressionfree survival [2-6]. In radiotherapy part, combination of whole pelvic radiotherapy (WPRT) and intracavitary brachytherapy (ICBT) has become a standard treatment modality for cervical cancer. Initial WPRT is performed to obtain tumor shrinkage to permit optical ICBT placement [7]. According to the American Brachytherapy Society recommendations for high-doserate (HDR) ICBT for carcinoma of the cervix, the whole pelvic external beam radiotherapy (EBRT) dose varies from institution to institution. The HDR fraction size and number depends on the EBRT dose [8]. HDR brachytherapy has been used for more than 30 years in Japan and Europe, and its applications have been increasing in the United States $[9,10]$. ICBT has been used to increase the dose to cervix to $\geq 80 \mathrm{~Gy}$. However organs in the proximity of the cervix, such as urinary bladder, small bowel and rectum, can receive rather high doses and adverse side effects can occur. The risk of late effects (i.e., obstruction, fibrosis/necrosis, or fistula) is related to the volume, total dose, dose per fraction, and specific intrinsic radiosensitivity of the normal tissue irradiated. Biologically effective dose (BED) had been used to evaluate the dose to target and organs at risk $[11,12]$. Because numerical values of BED (in $\mathrm{Gy}_{10} \mathrm{Or} \mathrm{Gy}_{3}$ ) are not familiar to many medical professionals, it is a common practice to convert these BEDs to the biologic equivalent of a total dose in 2 Gy fractions (BED of 2 Gy). These simplified biologically equivalent doses are called either EOD2, denoting equivalent total doses in 2-Gy fractions [13]. Rectal bleeding is a common late toxicity of pelvic radiation. Late toxicity to the rectum includes radiation proctitis, ulceration, stricture, and fistula. Some symptoms may resolve in a few months, whereas others will have a longer course that may involve 1 to 2 years of rectal bleeding. For severe cases, surgical intervention may be needed $[14,15]$.

To evaluate the rectal toxicity, many scores in objective and subjective approaches were used to evaluate. Radiation Therapy Oncology Group/European Organization for Research and Treatment of Cancer (RTOG/EORTC) toxicity scale were used in objective aspect only while Vienna Rectoscopy Score
(VRS) was used in subjective aspect $[16,17]$. Moreover, there were the combinations of subjective and objective evaluations as late effects of normal tissues (LENT-SOMA) scales [16] For this study, we retrospectively evaluated the endoscopic findings in cervical cancer patients who were treated by radical radiotherapy and also accumulative dose using EOD2 to rectum is associated with gastrointestinal toxicity $\geq$ grade 2 .

\section{Materials and Methods}

This study designed as retrospective study in 336 patients who were treated by radical radio-chemotherapy for locally advanced cervical cancer in the Division of Therapeutic Radiology and Oncology, Faculty of Medicine, Chiang Mai University during 2004-2009. We enrolled 39 patients of them who were evaluated the rectal toxicity by rectosigmoidoscopy during the follow-up period according to symptomatic indications or patient preferences. Treatment schedules for these patients were shown as the followings.

\section{External beam radiotherapy}

WPRT was performed using conventional radiotherapy with conventional fractionation (2 Gy/fraction) by 6- or 10 MV X-ray with two- or four-field techniques according to anteroposterior diameter. After 40 to 44 Gy of WPRT, central shielding was inserted using 3-or 4-cm width rectangular block at midline. The field was coned down after the initial 50 Gy in an effort to boost parametrium and exclude small bowel to $56 \mathrm{~Gy}$. For concurrent chemotherapy, mitomycin-C $10 \mathrm{mg} /$ $\mathrm{m}^{2}, 1 \mathrm{st}$ and 6 th week or weekly cisplatin $40 \mathrm{mg} / \mathrm{m}^{2}$ was given intravenously during WPRT [18].

\section{High-dose-rate brachytherapy}

First HDR-ICBT was started after 4th week of the treatment. Patients were treated with HDR-ICBT using Fletcher-type (Fletcher-Williamson Asian-Pacific) applicators. Anterior and posterior vaginal packing with gauze was used to maximize the distance from the source to the bladder wall and the rectal wall. The dose was prescribed to Point As with the dose of 6 to $7.2 \mathrm{~Gy}$ in 3 to 4 fractions. To evaluate rectal point dose, a rectal probe was used to identify the anterior rectal wall. The International Commission on Radiation Units and Measurements (ICRU) rectal points were identified using a laterally orthogonal radiographs taken during each individual application and defined as the points that an line drawn from the tip of ovoid applicator to the anterior border of the rectal 


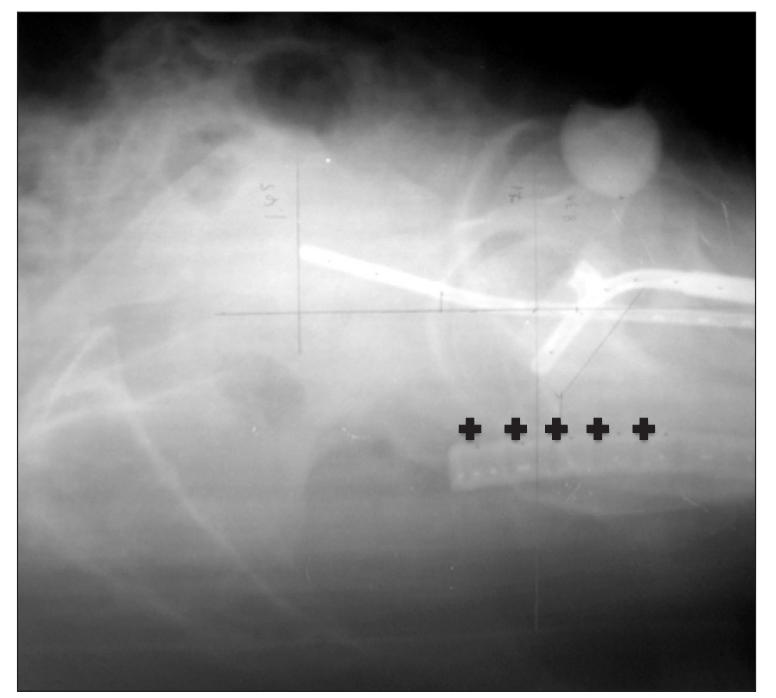

Fig. 1. Laterally orthogonal radiograph showed the points to evaluate rectal dose.

probe, the 4 another rectal points were digitized in 1- and 2-cm superior and inferior directions according to the first rectal point (Fig. 1). Calculation of the dose profiles was also based on these radiographs, and the maximum point dose was recorded.

\section{Calculation of rectal dose}

To determine the rectal dose from the WPRT plus ICBT, the total dose to rectal point was calculated as EQD2 using the linear quadratic model $[13,19]$.

\section{Follow-up schedule}

Late complications after RT are defined as side effects developing 3 months after RT completion. For each endpoint, the grade of late morbidity will be the maximal grade ever recorded, even if the side effect later subsided. The data will be retrospectively collected from treatment data and patient record of each patient. Patients that were sent to perform endoscopy were retrospectively collected to evaluate. The endoscopy report consists of a written description and a video photographic documentation. Rectal toxicity scoring is according to systematic sigmoidoscopy score for radiationinduced proctitis proposed by Medical University of Vienna using standard endoscopic terminology that had been previously used to evaluate the radiation-induced changes in the rectal mucosa of patients with prostate cancer treated with radical radiotherapy [17]. The grading system determines from the endoscopic terminology of the World Organization
Table 1. Patient characteristic data

\begin{tabular}{lc}
\hline \multicolumn{1}{c}{ Parameter } & Value \\
\hline Age (yr) & 50 (30-65) \\
Stage according to FIGO & 31 \\
IIB & 7 \\
IIIB & 1 \\
IVA & $76.4 \pm 5.8$ \\
EQD2 at point A (Gy) & 53.4 (IOR 44-64) \\
Total treatment time (day) & 58 (IOR 40-88) \\
Median endoscopic time (mo) & $64.2 \pm 9$ \\
Cumulative EQD2 at ICRU rectal point &
\end{tabular}

Values are presented as median (range) or mean \pm standard deviation.

FIGO, International Federation of Gynecology and Obstetrics; EQD2, equivalent dose of $2 \mathrm{~Gy}$; ICRU, International Commission on Radiation Units and Measurements; IQR, interquartile range.

for Digestive Endoscopy [20].

\section{Statistical analysis}

Observations will be censored at date of last visit, at 3 months of follow-up, or at date of failure. Logistic regression analysis was used to evaluate the association of cumulative rectal dose in EQD2 to toxicity. Descriptive statistics were used to evaluated the characteristic data of patients (age, treatment time, and etc.) All statistical analyses will be performed using IBM SPSS statistical software ver. 19.0 (IBM, Armonk, NY, USA).

\section{Results}

The mean cumulative dose in term of EQD2 to Point As and rectum were 76.4 and $64.2 \mathrm{~Gy}$, respectively. All Characteristic data of these patients were shown in Table 1.

\section{Toxicity profiles}

Grade 0-1 toxicity was observed in 34 patients for RTOG/ EORTC toxicity criteria and 25 patients for LENT-SOMA scale. In endoscopic findings, the first and second most common toxicities were congested mucosa (36 patients) and telangiectasia (32 patients). No necrosis was observed in this group. According to VRS proposed by Medical University of Vienna, grade 1 toxicity (51\%) was the most common finding. When we evaluated in VRS, there were 6, 20, 7, 3, and 3 patients for score $0,1,2,3$, and more than 3 , respectively. The data of toxicities were showed in Table 2.

When we considered the cumulative dose to rectum 
Table 2. Numbers of toxicity in patients recorded according to RTOG/EORTC toxicity scale, LENT-SOMA scales, and VRS

\begin{tabular}{lccccc}
\hline \multicolumn{1}{c}{ Toxicity scale } & Grade 0 or Score 0 & Grade 1 or Score 1 & Grade 2 or Score 2 & Grade 3 or Score 3 & More than 3 \\
\hline RTOG/EORTC toxicity scale & 22 & 12 & 5 & - & - \\
LENT-SOMA scale & 12 & 13 & 11 & 3 & - \\
VRS & 6 & 20 & 7 & 3 & 3 \\
\hline
\end{tabular}

RTOG/EORTC, Radiation Therapy Oncology Group/European Organization for Research and Treatment of Cancer toxicity scale; LENT-SOMA, late effects of normal tissues scales; VRS, Vienna Rectoscopy Score.

Table 3. Association between EQD2 and toxicity scale at least grade 2

\begin{tabular}{ccccc}
\hline Toxicity & Cumulative rectal dose in EQD2 (Gy) & No. of patients ${ }^{\text {a) }}(\mathrm{n}=39)$ & OR $(95 \% \mathrm{Cl})$ & $\mathrm{p}$-value \\
\hline LENT 2+ & $\leq 65$ & $4 / 21$ & 1 & 0.022 \\
\multirow{2}{*}{ RTOG 2+ } & $>65$ & $10 / 18$ & 1 & $0.312(1.269-22.244)$ \\
VRS 2+ & $\leq 65$ & $1 / 21$ & $5.174(0.576-56.727)$ \\
& $>65$ & $4 / 18$ & 1 & 0.497 \\
& $\leq 65$ & $6 / 21$ & $1.591(0.417-6.073)$
\end{tabular}

EQD2, equivalent dose of 2 Gy; LENT-SOMA, late effects of normal tissues scales; RTOG, Radiation Therapy Oncology Group; VRS, Vienna Rectoscopy Score; $\mathrm{OR}$, odds ratio; $\mathrm{Cl}$, confidence interval.

a) indicates the number of patients who developed at least grade 2 toxicity/the number of patients who had the cumulative rectal dose.

and toxicity scale, we found the associations between the cumulative dose to rectum more than 65 Gy and LENT-SOMA scale at least grade 2 ( $p=0.022$; odds ratio, 5.312; 95\% confidence interval, 1.269-22.244). When we evaluated the association of the same dose level (>65 Gy) and other toxicity scale, No association was found in RTOG/EORTC ( $p=0.137)$ and VRS ( $p=0.497)$ (Table 3).

\section{Discussion and Conclusion}

During the past decade, BED was used to evaluate the cumulative dose to tumor and organs-at-risk. For cervical cancer, the cumulative dose to rectum and bladder in terms of BED correlated to rectal toxicity $[21,22]$.

Rectal toxicity is the most common toxicity after radical radiotherapy for cervical cancer. The combined dose of EBRT and brachytherapy to rectum predicted the toxicity in terms of BED. Toita et al. [23] suggested that cumulative BED at the rectal point should be kept below $100-120 \mathrm{~Gy}_{3}$ to prevent late rectal complication. The supported study from Huang et al. [24] and Suzuki et al. [25] reported significant higher rectal toxicity in patients that cumulative rectal BED $\geq 100 \mathrm{~Gy}_{3}$. Moreover, Ogino et al. [26] also showed that the time-dose factor and the BED at rectal point values were significant correlated with the incidence of late rectal complication.

In terms of EQD2, Sakata et al. [27] found that rectal radiation tolerance dose using EQD2, 5\% and 50\% complication probability, was about 64 and $79 \mathrm{~Gy}$, respectively, and the probability of rectal complications increased drastically after the maximal rectal dose was $>60 \mathrm{~Gy}$.

From our study, only association between cumulative rectal dose in EQD2 >65 Gy and LENT-SOMA $\geq 2$ were observed ( $p=$ 0.022). No statistical significance was found between rectal dose and toxicity in terms of RTOG/EORTC scale $(p=0.137)$ and VRS ( $p=0.497)$. The possible explanation is that LENT-SOMA scale covered both objective and subjective evaluations while RTOG/EORTC and VRS were purely objective and subjective approaches, respectively. So LENT-SOMA scale should be more appropriate to evaluate the rectal toxicity. Moreover, in this study, the association of cumulative rectal toxicity in EQD2 to toxicity $\geq 1$ was not found statistical significance in all toxicity score. This correlates to the clinical aspects that the decision to treat toxicity starts practically when patients developed $\geq$ grade 2 toxicity [28].

However, there are some limitations in this study. The accuracy of the rectal dose calculation is affected by several factors. First, to calculate the rectal dose from EBRT, It assumed that the rectum was completely shielded after central shielding. However, the rectum might be irradiated with some doses according to anatomic variations. Secondly, using orthogonal films for calculation might cause problem to evaluate the really maximal dose to rectum due to anatomy. Volume-based 
planning brachytherapy could be more accurate on delineating organs-at-risk including rectum and assessing doses through dose volume histograms [29]. The study from Kato et al. [30] reported the dose evaluation of 48 patients who were treated by combination of WPRT plus HDR-ICBT. Computed tomography-based three dimensional (3D) dose-volume parameters of the rectum may be effective for predicting late rectal complications while no correlation between rectal dose in ICRU point and toxicity was found [30]. The using of 3D-based approach will be reported in the future. Thirdly, the number of patients who performed endoscopic exam in our study was small because rectosigmoidoscopy was not routine evaluation after treatment in our country.

Our findings showed correlation between cumulative EQD2 to rectal point $>65$ Gy and rectal toxicity in term of LENT-SOMA scale at least grade 2 . The volume-based planning in both WPRT and ICBT improve the accuracy in dosimetry and toxicity evaluation.

\section{Conflict of Interest}

No potential conflict of interest relevant to this article was reported.

\section{Acknowledgments}

This work was supported by the National Research University (NRU) Project under Thailand's Office of the Higher Education Commission for financial support. The author offers many thanks to the staffs of the NRU-CMU in the Gynecologic Oncology Cluster and the Division of Therapeutic Radiology and Oncology, Faculty of Medicine, Chiang Mai University for supporting this study.

\section{References}

1. Khuhaprema $T$, Srivatanakul $P$, Attasara $P$, Sriplung $H$, Wiangnon S, Sumitsawan Y. Cancer in Thailand (volume V, 2001-2003). Bangkok: Ministry of Public Health and Ministry of Education; 2010.

2. Morris M, Eifel PJ, Lu J, et al. Pelvic radiation with concurrent chemotherapy compared with pelvic and para-aortic radiation for high-risk cervical cancer. N Engl J Med 1999;340:1137-43.

3. Whitney CW, Sause W, Bundy BN, et al. Randomized comparison of fluorouracil plus cisplatin versus hydroxyurea as an adjunct to radiation therapy in stage IIB-IVA carcinoma of the cervix with negative para-aortic lymph nodes: a Gynecologic
Oncology Group and Southwest Oncology Group study. J Clin Oncol 1999;175:1339-48.

4. Rose PG, Bundy BN, Watkins EB, et al. Concurrent cisplatinbased radiotherapy and chemotherapy for locally advanced cervical cancer. N Engl J Med 1999;340:1144-53.

5. Monk BJ, Tewari KS, Koh WJ. Multimodality therapy for locally advanced cervical carcinoma: state of the art and future directions. J Clin Oncol 2007;25:2952-65.

6. Eifel PJ. Chemoradiotherapy in the treatment of cervical cancer. Semin Radiat Oncol 2006;16:177-85.

7. National Comprehensive Cancer Network. NCCN Guidelines: cervical cancer screening (ver. 1.2011). Washington, PA: National Comprehensive Cancer Network; 2010.

8. Nag S, Erickson B, Thomadsen B, Orton C, Demanes JD, Petereit D. The American Brachytherapy Society recommendations for high-dose-rate brachytherapy for carcinoma of the cervix. Int J Radiat Oncol Biol Phys 2000;48:201-11.

9. Nag S, Orton C, Young D, Erickson B. The American Brachytherapy Society survey of brachytherapy practice for carcinoma of the cervix in the United States. Gynecol Oncol 1999;73:111-8.

10. Erickson-Whitmann B, Rownd J, Khater K. Biologic and physical aspects of radiation oncology. In: Barakat RR, Markman $M$ Randall ME, editors. Principles and practice of gynecology oncology. 5th ed. Philadelphia, PA: Lippincott Williams \& Wilkins; 2009. p. 325-80.

11. Kong FM, Pan C, Eisbruch A, Ten Haken RK. Physical models and simpler dosimetric descriptors of radiation late toxicity. Semin Radiat Oncol 2007;17:108-20.

12. Fowler JF. Brief summary of radiobiological principles in fractionated radiotherapy. Semin Radiat Oncol 1992;2:16-21.

13. Joiner MC, Bentzen SM. Time-dose relationships: the linearquadratic approach. In: Steel GG. Basic clinical radiobiology. 3rd ed. London: Oxford University Press; 2002. p. 120-33.

14. Jackson A. Partial irradiation of the rectum. Semin Radiat Oncol 2001;11:215-23.

15. Coia LR, Myerson RJ, Tepper JE. Late effects of radiation therapy on the gastrointestinal tract. Int J Radiat Oncol Biol Phys 1995;31:1213-36.

16. Pavi J, Denekamp J, Letschert J. LENT SOMA scales for all anatomic sites. Int J Radiat Oncol Biol Phys 1995;31:1049-91.

17. Wachter S, Gerstner N, Goldner G, Potzi R, Wambersie A, Potter R. Endoscopic scoring of late rectal mucosal damage after conformal radiotherapy for prostatic carcinoma. Radiother Oncol 2000;54:11-9.

18. Kamnerdsupaphon $P$, Chitapanarux I, Sukthomya V, Lorvidhaya

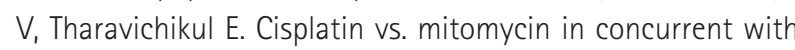
radiotherapy for locally advanced cervical cancer. J Thai Soc Ther Radiol Oncol 2008;14:61-70.

19. Fowler JF. The linear-quadratic formula and progress in fractionated radiotherapy. Br J Radiol 1989;62:679-94. 
20. Crespi $M$, Delvaux $M$, Schaprio $M$, Venables $C$, Zwiebel $F$. Working Party Report by the Committee for Minimal Standards of Terminology and Documentation in Digestive Endoscopy of the European Society of Gastrointestinal Endoscopy. Minimal standard terminology for a computerized endoscopic database. Ad hoc Task Force of the Committee. Am J Gastroenterol 1996;91:191-216.

21. Kim TH, Choi J, Park SY, et al. Dosimetric parameters that predict late rectal complications after curative radiotherapy in patients with uterine cervical carcinoma. Cancer 2005;104: 1304-11.

22. Chen SW, Liang JA, Yang SN, Liu RT, Lin FJ. The prediction of late rectal complications following the treatment of uterine cervical cancer by high-dose-rate brachytherapy. Int J Radiat Oncol Biol Phys 2000;47:955-61.

23. Toita $T$, Kakinohana $Y$, Ogawa $K$, et al. Combination external beam radiotherapy and high-dose-rate intracavitary brachytherapy for uterine cervical cancer: analysis of dose and fractionation schedule. Int J Radiat Oncol Biol Phys 2003;56: 1344-53.

24. Huang EY, Wang CJ, Hsu HC, Hao Lin, Chen HC, Sun LM. Dosimetric factors predicting severe radiation-induced bowel complications in patients with cervical cancer: combined effect of external parametrial dose and cumulative rectal dose. Gynecol Oncol 2004;95:101-8.
25. Suzuki O, Yoshioka Y, Isohashi F, et al. Effect of high-dose-rate 192Ir source activity on late rectal bleeding after intracavitary radiation therapy for uterine cervix cancer. Int J Radiat Oncol Biol Phys 2008;71:1329-34

26. Ogino I, Kitamura T, Okamoto $N$, et al. Late rectal complication following high dose rate intracavitary brachytherapy in cancer of the cervix. Int J Radiat Oncol Biol Phys 1995;31:725-34.

27. Sakata K, Nagakura H, Oouchi A, et al. High-dose-rate intracavitary brachytherapy: results of analyses of late rectal complications. Int J Radiat Oncol Biol Phys 2002;54:1369-76.

28. Cox JD, Stetz J, Pajak TF. Toxicity criteria of the Radiation Therapy Oncology Group (RTOG) and the European Organization for Research and Treatment of Cancer (EORTC). Int J Radiat Oncol Biol Phys 1995:31:1341-6.

29. Koom WS, Sohn DK, Kim JY, et al. Computed tomographybased high-dose-rate intracavitary brachytherapy for uterine cervical cancer: preliminary demonstration of correlation between dose-volume parameters and rectal mucosal changes observed by flexible sigmoidoscopy. Int J Radiat Oncol Biol Phys 2007;68:1446-54.

30. Kato S, Tran DN, Ohno T, et al. CT-based 3D dose-volume parameter of the rectum and late rectal complication in patients with cervical cancer treated with high-dose-rate intracavitary brachytherapy. J Radiat Res 2010;51:215-21. 